\title{
Management accounting in the context of digitalization
}

\author{
Alexey Platov ${ }^{1, *}$, Munir Kalemulloev ${ }^{2}$, and Shakhlo Zikirova ${ }^{1}$ \\ ${ }^{1}$ Moscow (Senkevich) State Institute of Physical Culture, Sports and Tourism, 125499 Moscow, \\ Russia \\ ${ }^{2}$ Tajik National University, 73402517 Dushanbe, Tajikistan
}

\begin{abstract}
This article examines aspects of digitalization as a way to optimize management accounting and control the movement of inventory of enterprises. Revealed the advantages that digitalization provides for management accounting at the enterprise. The stages of the process of digitalization of management accounting of an enterprise are described. Within the framework of the process approach, an algorithm for forming a digital model of an effective management accounting process is constructed. On the basis of the proposed algorithm, a digital model of the process of management accounting and control of the movement of inventories has been developed. The model assumes monitoring of management and supporting processes, and also contains a mechanism for continuous monitoring of compliance with the goals of the enterprise and the absence of bottlenecks. The increase in the efficiency of management accounting and control of the movement of stocks and management of the enterprise as a whole, provided by digitalization and the use of a process approach, is emphasized.
\end{abstract}

\section{Introduction}

Digitalization is the process of transitioning to digital business. It is characterized by the use of digital technologies that change business models and provide new organizational opportunities [1]. Digitalization has affected all types of business activities, from business models to support functions such as management accounting. Digitization has fostered new forms of collaboration between companies, suppliers, customers and employees, resulting in new products and services. At the same time, digitalization remains a challenge for most companies, as it requires them to rethink their strategy and analyze new business opportunities. In the financial sector, digitalization has created conditions for the automation and robotization of routine processes, the introduction of business intelligence and data analytics. Digital technologies are transforming and expanding the types and sources of data used in management accounting. They are also transforming important accounting processes [2].

The first phase of digitalization, which significantly changed the way organizations and management accountants operate, was the emergence of computerized information systems

\footnotetext{
* Corresponding author: aplatov@yandex.ru
} 
$[3,4]$. In the 1960s and 1970s, such systems allowed management accountants to record data in greater detail and conduct more accurate analysis. The second phase came with the spread of the Internet and IIS [4]. In this context, IIS can be considered management accounting systems [5]. The third phase of digitalization is currently developing. The emergence of a whole range of new information technologies has a strong impact on the business processes of organizations, including management accounting [6, 7].

In the new environment, in order to maintain its viability, the organization must develop a digital strategy. Such a strategy takes into account the benefits of digital technologies, indicating the direction of development. It enables managers to lead digital initiatives and measure their progress [8].

Today, management accountants need to develop new digital competencies without losing traditional ones $[9,10]$. At the organizational level, financial business processes are likely to face staff cuts [11]. However, this does not diminish the importance of the finance function; on the contrary, both new opportunities and new roles will appear [10].

Over the past few years, academics and practitioners have noted a fundamental transformation in management accounting due to digitalization and growing globalization [12]. It has been found that CFOs and management accountants are all more likely to expect a change in finance function in their company. However, most finance departments in large companies have made little progress in their digitalization efforts despite expectations of change [13] (Schäffer \& Weber, 2018b). Half of the management accountants indicated that their company did not have a digitalization strategy for accounting, a third reported having only a preliminary strategy, and only $6 \%$ of companies had such a strategy. Kolthof et al.found that robotic process automation (RPA) is used to process transactions in less than half of the operations [9].

According to Schäffer and Weber, adaptation processes took longer than the management accountants originally anticipated [14].

Today, issues of digitalization of management accounting and control of the movement of inventory items at enterprises attract close attention of practitioners and the scientific community. We define digital transformation as the process of forming new business and operating models by creating a basis for the implementation and integration of digital technologies. The goal of digitalization is to achieve new organizational capabilities by digitally transforming operational processes and interactions. Digital transformation is not only and not so much the introduction of new software, but mainly the use of it as an element of a strategy and a holistic digital appeal to people, processes and technologies.

The main advantages of digitalization of management accounting for an organization, in our opinion, are:

- promoting the creation of new organizational capabilities, increasing the efficiency and flexibility of the enterprise;

- automation and acceleration of the circulation of inventory items;

- ensuring uninterrupted interaction of structural units with supply chains as a whole;

- the possibility of deeper data analysis, which improves the quality of interactions both within the enterprise and with suppliers;

- the ability of employees to focus on innovation and creativity, rather than on routine operations. The main advantages of digitalization of management accounting for an organization, in our opinion, are:

- promoting the creation of new organizational capabilities, increasing the efficiency and flexibility of the enterprise;

- automation and acceleration of the circulation of inventory items;

- ensuring uninterrupted interaction of structural units with supply chains as a whole;

- the possibility of deeper data analysis, which improves the quality of interactions both within the enterprise and with suppliers; 
- the ability of employees to focus on innovation and creativity, rather than on routine operations.

\section{Methods}

Digital transformation for most organizations and their structural units requires the formation of a new model. In particular, improving the efficiency of the inventory management process requires the use of a new generation operating model. This model is an innovative management approach that integrates digital technology and operational capabilities in an integrated, sequential manner to achieve incremental improvements in management, accounting, and control efficiency.

We see the digitalization of management accounting in the implementation of the following stages (Fig. 1). From the standpoint of managing and controlling the movement of inventory and supply chains, digital transformation is often associated with the Gartner Stage maturity model [14. This model claims that the level of development of a process is determined by the degree of its controllability, as well as the ability to control and monitor.

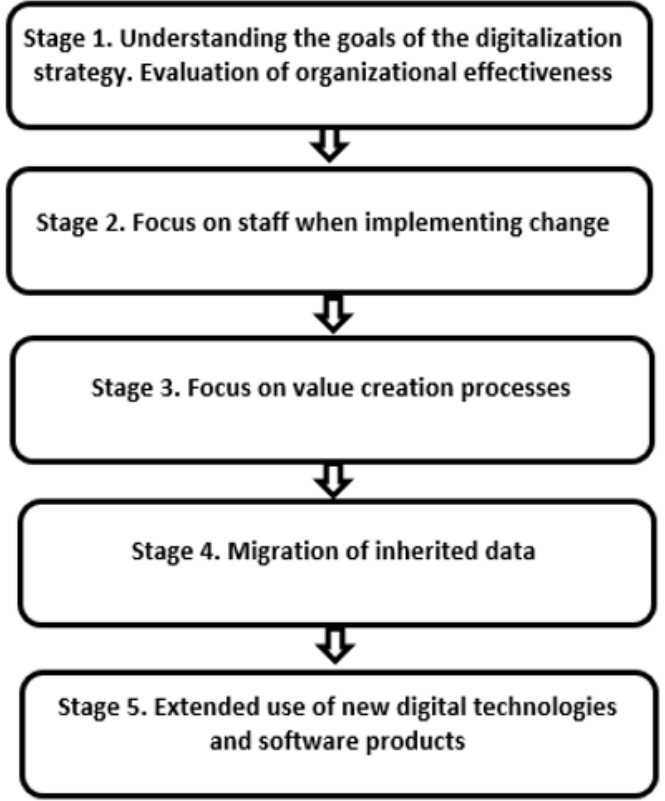

Fig. 1. Stages of digitalization of management accounting of the enterprise.

Upon reaching the next level of development, the indicators of management efficiency increase, which leads to a decrease in deviations between the target and actual values of performance indicators. Each level is the basis for a more rational and efficient implementation of the processes of control over the movement of inventories and supplies at the next levels, although counterparties can use the methods and technologies recommended for the processes of higher levels. Without the use of digital technologies, it is impossible to quickly track the requests of individual structural divisions, the availability of the necessary inventory items in the warehouse and the need to organize regular deliveries. It is the use of digital technologies and appropriate management systems that ensures the speed of decisionmaking based on current information, the ability to foresee the future need for commodity and material values. 


\section{Results and discussion}

Most often, the digital transformation of the supply chain disrupts its operations, but in the long term it protects against much more serious disruptions. Technologies that lead to transformation include blockchain, the Internet of Things, big data analytics, chatbots, lead to the integration of data by counterparties in the supply chain, and better interaction with consumers. The big data ecosystem is the foundation for the digital transformation of inventory control. It begins with the proliferation of sensors that transmit data over the Internet to a cognitive computing platform. Such a platform provides analysis and interpretation of information necessary for making informed management decisions. The specific composition of digital technologies used at the enterprise depends on many factors, primarily on the scope of the economic activity of the enterprise. The activities of enterprises, especially industrial ones, are accompanied by the need to process a significant number of documents containing purchase agreements, purchase orders, delivery data, and the like. Much of this work can be done electronically, which is why consultants are increasingly recommending the use of digital technology when controlling the movement of inventory. In addition, digitalization means a complete paradigm shift in organizational culture and operations, since it is necessary not only to acquire the latest digital tools, but also to create a new management system, a new business model, new thinking.

It is possible to identify certain stages of the digital transformation of an enterprise's activities, in particular, the digitalization of management accounting and control over the movement of inventory items. Figure 2 illustrates the stages of digital transformation of the processes of management and control of the movement of inventories - from the Internet of Things and Blockchain technologies to Artificial Intelligence, which ensures the adoption of optimal management decisions in the process of management, accounting and control of the movement of inventories in the absence of direct involvement of employees.

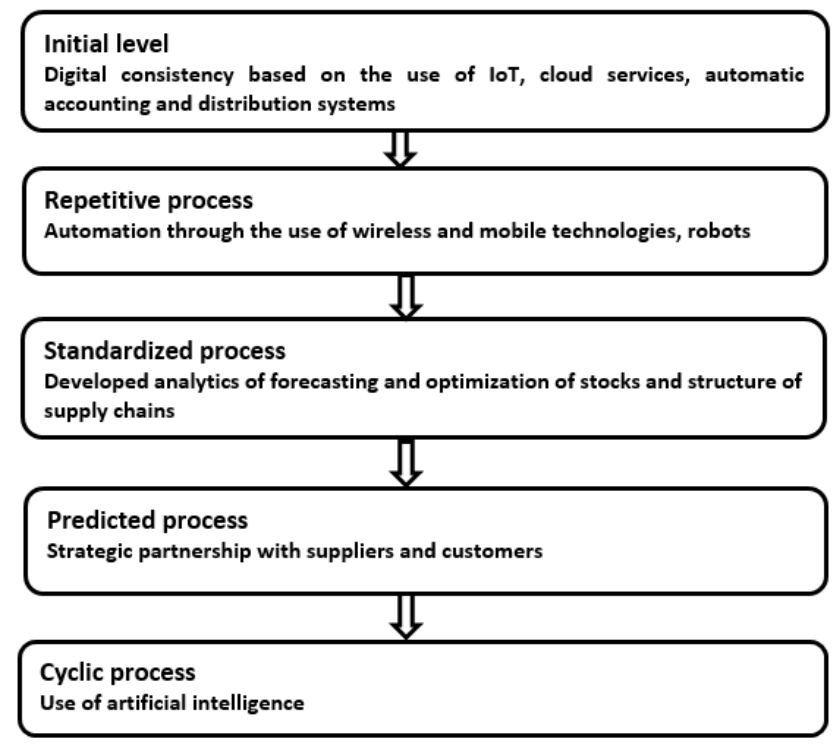

Fig. 2. Stages of digital transformation of processes of management and control of the movement of inventories.

At the same time, it should be noted that digitalization when using the process approach, increasing the efficiency of management accounting and control over the movement of inventories and enterprise management as a whole, is also accompanied by significant risks. In particular, these risks are: 
- the risk of incorrect or incomplete display of information associated with insufficient qualifications or dishonesty of employees as a result of compliance with unintentional or deliberate errors;

- the risk of losing information due to insufficient protection of the system from external influences (hacker attacks);

- the risk of interruptions (downtime) in the business of the enterprise due to temporary interruptions in the operation of software, equipment of Internet providers.

In accordance with the international quality standards of the ISO series, the process approach is a priority in enterprise management. Management and control of the movement of inventory should be considered as one of the processes that ensures the activities of the enterprise as a whole. Figure 3 shows the algorithm for forming a model of an effective process of management and control of the movement of inventory.

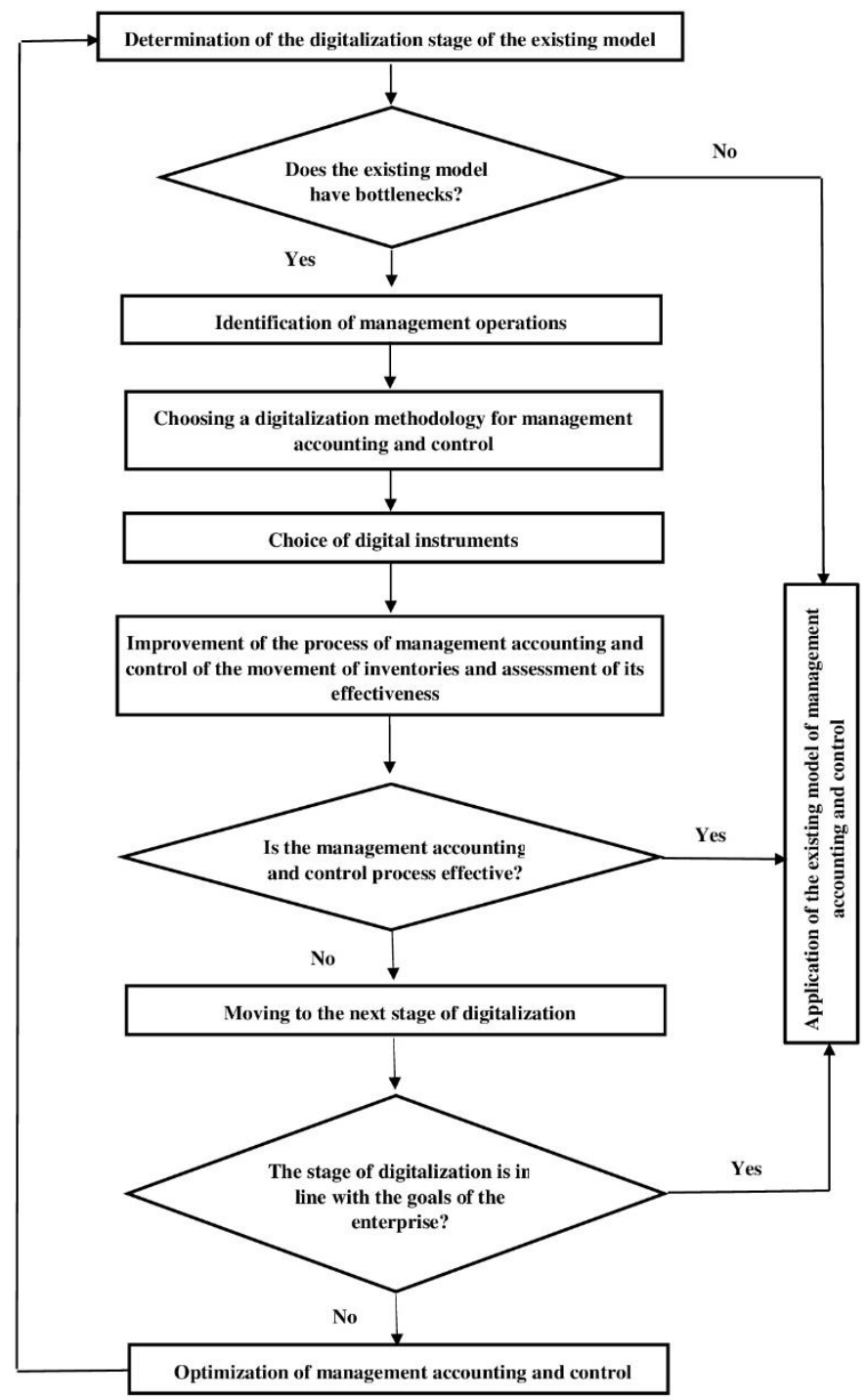

Fig. 3. Stages of digital transformation of processes of management and control of the movement of inventories. 
The use of a process approach and digital transformation will make it possible to automatically record the receipt, movement, use and sale of inventory, to monitor compliance with the availability of inventory to the minimum allowable current stock. The system, having found that the level of inventory has decreased to the minimum allowable, must send the appropriate notification to the manager of the enterprise, which will decide to place an extraordinary order or replenish the stock from other sources. In general, the scheme of the proposed process model of management and control of the movement of inventory of the enterprise is shown in Figure 4.

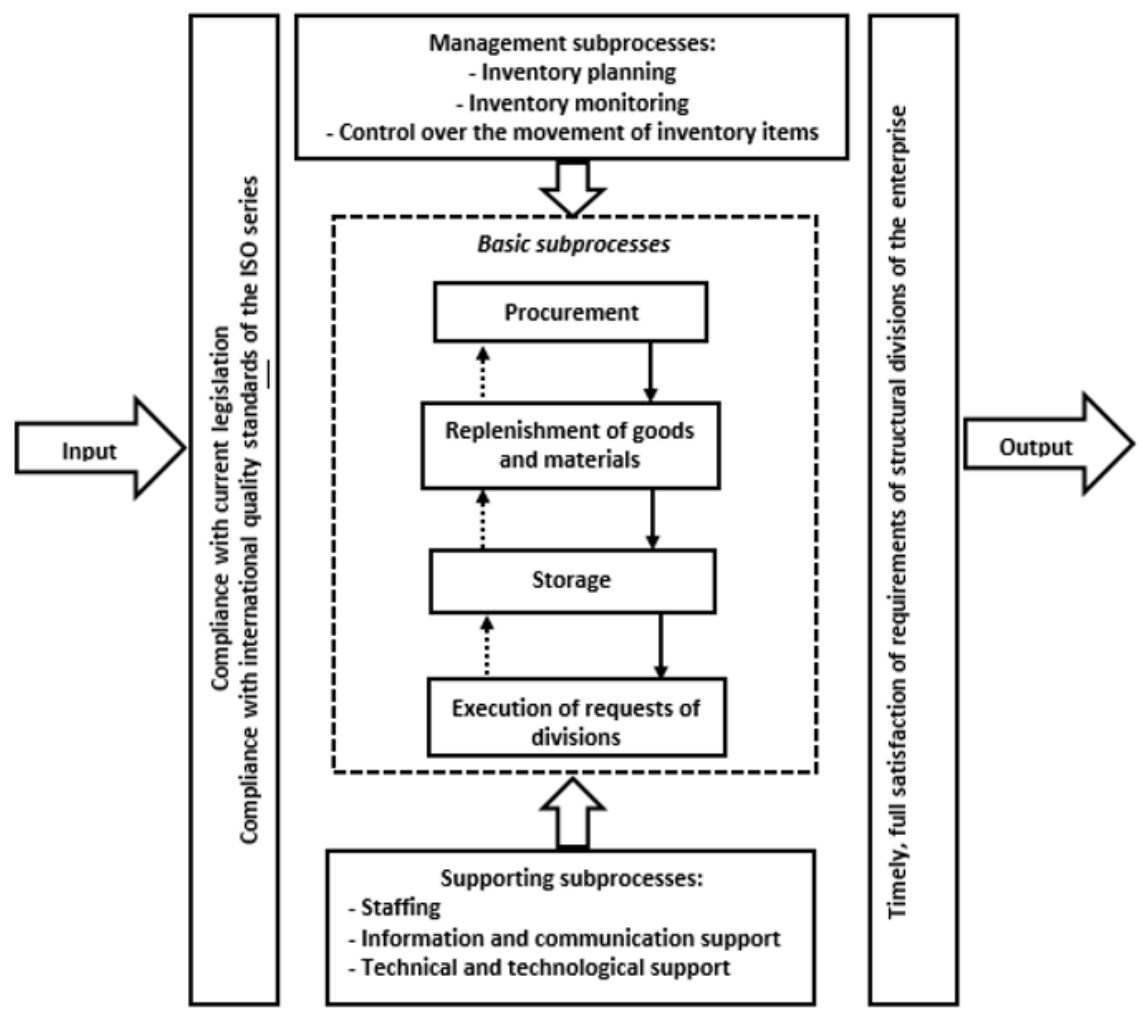

Fig. 4. Model of effective management accounting process based on digitalization.

The proposed model of management and control of the movement of inventory is formed on the basis of a process approach, which involves not only the study of management and support processes, but also constant verification of the model for compliance with the objectives of the enterprise and the absence of "bottlenecks".

\section{Conclusion}

Digitalization in modern conditions is an objective phenomenon that accompanies the formation of the information economy and globalization processes. Digitalization of economic activity and, in particular, management accounting and control of the movement of inventory leads to such benefits as: the creation of new organizational opportunities, increasing the efficiency and flexibility of activities; automation and acceleration of turnover of inventory; ensuring uninterrupted interaction of structural units with supply chains in general; the ability of employees to focus on innovation and creativity, rather than routine operations; possibility of deeper data analysis; improving the quality of interaction both within the 
enterprise and with suppliers. At the same time, the digital transformation leads to additional risks of economic activity (risk of incorrect or incomplete reflection of information, risk of information loss, risk of downtime).

Provided that risks are taken into account, preventive measures are introduced in order to prevent them or minimize them, digitalization in general provides an increase in the efficiency of the enterprise. In addition, digitalization is a phenomenon that covers almost all areas of socio-economic activity. Given this, the lag in the digital transformation of economic activity of the enterprise at present actually means the loss of significant competitive advantages.

\section{References}

1. D.-R. Knudsen, Int. J. Account. Inf. Syst., 36 (2020)

2. M. Arnaboldi, G. Azzone, Y. Sidorova, Account. Audit. Account. J., 30 (4), 821 (2017)

3. M. Granlund, J. Mouritsen, Eur. Account. Rev., 12 (1), 77 (2003)

4. M. Porter, J. Heppelmann, Harv. Bus. Rev., 92 (2014)

5. A. Rom, C. Rohde, Int. J. Account. Inf. Syst., 8 (1), 40 (2007)

6. J. Karimi, Z. Walter, J. Manag. Inf. Syst., 32 (1), 39 (2015)

7. P. Parviainen, M. Tihinen, J. Kääriäinen, S. Teppola, Int. J. Inf. Syst. Proj. Manag., 5(1), 63 (2017)

8. J. Ross, C. Beath, I. Sebastian, MIT Sloan Manag Rev, 58(2),7 (2017)

9. E. Kolthof, E. Steenwijk, F. Verbeeten, MCA, 6, 16 (2017)

10. U. Schäfer, L. Brueckner, CMR, 63(7), 14 (2019)

11. C. Frey, M. Osborne,. Technol Forecast Soc Change, 114, 254 (2017)

12. U. Schäfer, J. Weber, CMR, 60 (6), 8 (2016)

13. U. Schäfer, J. Weber, Frankfurter Allgemeine Zeitung, 16, March 26.

14. U. Schäfer, J. Weber, Behavioral Controlling: Anniversary Volume in Honor of Jürgen Weber, 179-190 (2019) 